\title{
NK/T cell Lymphoma as a Rare Cause of an Oronasal Fistula
}

\section{Ahmad Izani Mohd Safian', [MD] ORCID: 0000-0001-7677-6356}

Ahmad Fakrurrozi Mohamad², [MD] ORCID: 0000-0002-5916-8444

Ramiza Ramza Ramli', [MD] ORCID: 0000-0001-8196-321X

Irfan Mohamad', [MD]

ORCID: 0000-0001-8572-0514

The study has not been presented previously in a congress or symposium.

'Department of Otorhinolaryngology-Head and Neck Surgery, Health Campus, School of Medical Sciences, Universiti Sains Malaysia, Kubang Kerian, Kelantan, Malaysia.

${ }^{2}$ Department of Oromaxillofacial Surgery, Health Campus, School of Medical Sciences, Universiti Sains Malaysia, Kubang Kerian, Kelantan, Malaysia.

Corresponding Author: Irfan Mohamad Department of Otorhinolaryngology-Head and Neck Surgery, Health Campus, School of Medical Sciences, Universiti Sains Malaysia, Kubang Kerian, Kelantan, Malaysia.

E-mail: irfankb@usm.my

\section{sel ABSTRACT Cen}

NK/T cell lymphoma is one of the most unique and rare forms of extranodal non-Hodgkin's lymphoma, mostly derived from natural killer cell lineages and occasionally cytotoxic $T$ cell lines. Due to the nonspecific presentation of NK/T cell lymphoma such as nasal obstruction, nasal discharge, and epistaxis, diagnosis is often an issue and can be misleading. Oronasal fistula following a non-healing ulcer on the soft palate, can be one of the clinical presentations of NK/T cell lymphoma. Here, we are reporting a rare case of NK/T cell lymphoma in a 32-year-old gentleman who presented with an oronasal fistula post tissue biopsy for a non-healing ulcer over the soft palate. The tissue biopsy of the soft palate mass was revealed as NK/T cell lymphoma and was staged as Stage $1 \mathrm{~b}$ after computed tomographic imaging revealed a local tumour invasion without sign of nodal involvement and no metastasis. The patient eventually started with the SMILE Protocol and responded well up to the date. The ulcer healed but the fistula persisted. Primary closure was planned by the oromaxillofacial surgery team after completion of chemotherapy.

Keywords: Hematological disorder, oncology, oronasal fistula

https://doi.org/10.32552/2021.ActaMedica.544

Received: 6 December 2020, Accepted: 22 September 2021

Published online: 21 November 2021

\section{INTRODUCTION}

Extranodal NK/T cell lymphoma (NKTCL) nasal type or previously known as lethal midline granuloma, is considered as one of the rarest differential diagnoses to cause an oronasal fistula (ONF). It is a belligerent malignancy of putative NK-cell origin, with a minority deriving from the T-cell lineage. About $80 \%$ of cases occur in the nose, nasopharynx, oropharynx, the Waldeyer ring, and parts of the upper aerodigestive tract, while $20 \%$ in non-nasal areas such as skin, gastrointestinal tract, testis, salivary gland, etc [1].
ONF is an internal fistula defined by an abnormal epithelialized track communicating the nasal cavity with the oral cavity in which it may be due to multiple causative factors and can be divided into congenital, infection, iatrogenic, and tumour causes. Tumours such as squamous cell carcinoma, adenoid cystic carcinoma, melanoma, acute lymphoblastic leukemia and lymphoma are known causes of ONF. Nevertheless, extranodal NKTCL itself is a rare encounter [2]. 
Symptoms of the NKTCL nasal type are varied and non-specific. It may include nasal discharge, nasal obstruction, epistaxis, and other sinonasal symptoms. Thus, it is frequently missed and treated as a viral or bacterial infection, which leads to a late diagnosis and increases morbidity and mortality. Therefore, an early diagnosis of the disease has to be made meticulously [3].

\section{CASE PRESENTATION}

A 32-year-old man with no known medical illness, presented with a two-day history of left epistaxis. It was associated with worsening bilateral nasal blockage, rhinorrhea and hyposmia for six months. He also complained of a painless 'hole' on the left side of his soft palate which started as an ulcer and has subsequently increased in size for the past three months. It was complicated by nasal regurgitation as well as hypernasal voice. The nasal regurgitation was reduced by taking food in small amounts while the epistaxis was minimal in amount and had resolved spontaneously.

The patient had an intermittent low-grade fever with night sweats and a significant weight loss of 20 kilograms in the past two months. He denied having any symptoms or contact with tuberculosis patients. He did not have odynophagia, dysphagia, hypernasality voice, stridor, shortness of breath, or neck swelling. There were no other complaints except for reduced hearing, especially over the left ear.

On examination, he was a moderately-built adult with good hydration, not anaemic nor septic looking. Vital signs were within normal limits with no signs of anaemia. He had no palpable cervical, axillary or inguinal lymph nodes. All cranial nerves were grossly intact except for the reduced sensation of smell in bilateral nostrils.

An intraoral examination revealed an irregular edge fistula over the left side of the soft palate connecting the oral and nasal cavity, measuring $3 x$ $3 \mathrm{~cm}$ (Figure 1). Upon palpation, the area was slightly tender but with no contact bleeding. Otherwise, the surrounding structures, including the bilateral tonsils, uvula and anterior pillars, were normal. The nasal endoscopy showed a fleshy mass occupying the left nasal cavity (Figure 2). His right nasal airway had a hypertrophied inferior turbinate with no mass seen, with the septum intact. Otoscopy showed a retracted left tympanic membrane with evidence of conductive hearing loss.

The laboratory test showed normal findings except for his lactic acid dehydrogenase (LDH) level, which was high at 419 units per liter (U/L). His EBV PCR was reported as positive (POS 2.7 LOG IU).

Biopsy of the left soft palate ulcer revealed lymphoproliferative neoplasm of T-cell lineage with a high proliferation index. Bone marrow aspiration revealed no infiltration. The Contrast-enhanced computed tomography (CECT) paranasal sinus showed focal irregularities in the soft palate with no

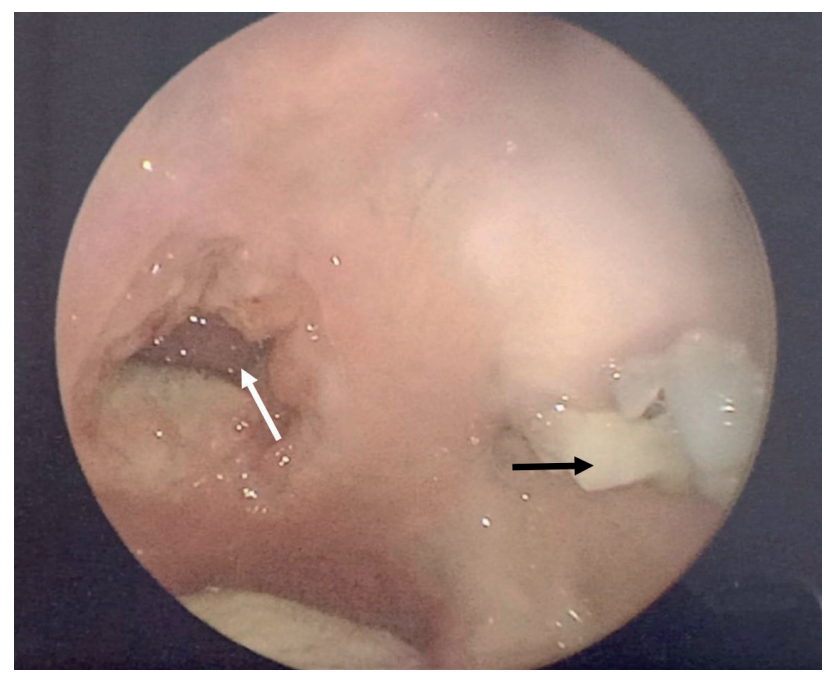

Figure 1. Rough-edged, single non-healing ulcer over the left soft palate (white arrow), creating a fistula with the left nasal cavity. There is a partially erupted left upper third molar tooth (black arrow).

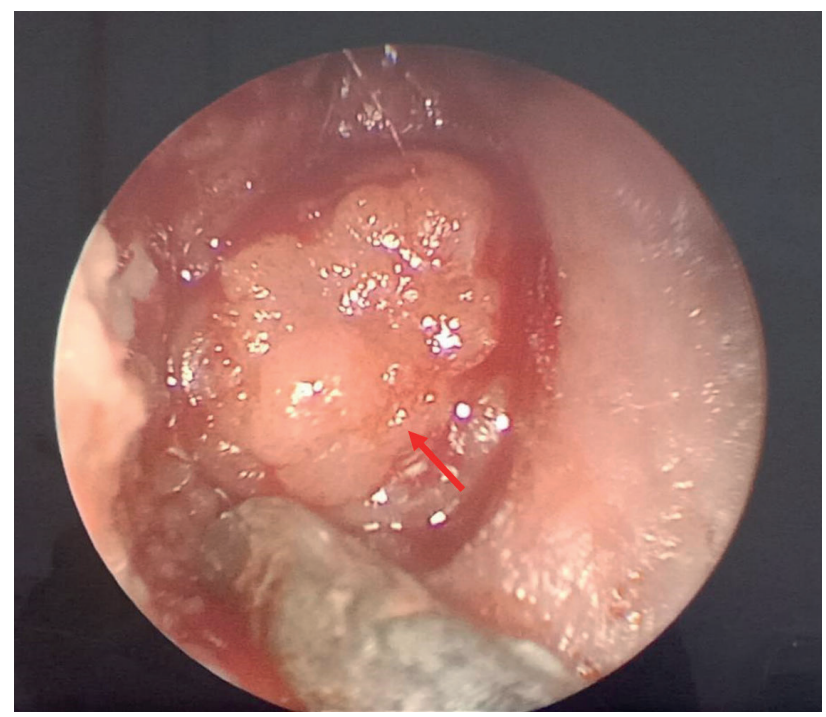

Figure 2. Left nasal endoscopy showed an irregularsurfaced fleshy mass occupying left anterior nasal cavity with minimal bleeding (red arrow). 
focal enhancing lesion seen. An apparent exophytic hypodense lesion in the superior aspect of the soft palate which is projecting into the nasopharynx with the soft palate and uvula appearing bulky and irregular (Figure 3). There were subcentimeter cervical nodes seen with hepatosplenomegaly.

A final diagnosis of NKTCL, Stage $1 \mathrm{~b}$ (T2NOMO) was made and the patient is currently undergoing chemotherapy (SMILE Protocol). Besides the ongoing therapy, the patient was given chlorhexidine mouth wash $10 \mathrm{ml}$ TDS with an oral moisturizer to keep a normal oral $\mathrm{pH}$, therefore avoiding dental carries. On day 19 of his chemotherapy, the size of the ONF widened to $5 \mathrm{~cm} \times 4 \mathrm{~cm}$, but with more healthy-looking edges (Figure 4). The patient was planned for surgical closure of the ONF after completion of the chemotherapy. If surgical closure fails, a maxillary obturator prosthesis may be applied.

\section{DISCUSSION}

In this case, ONF developed following a persistent non-healing ulcer on the left soft palate and had worsened post biopsy of the ulcerated part. ONF is an abnormal epithelial communicating tract

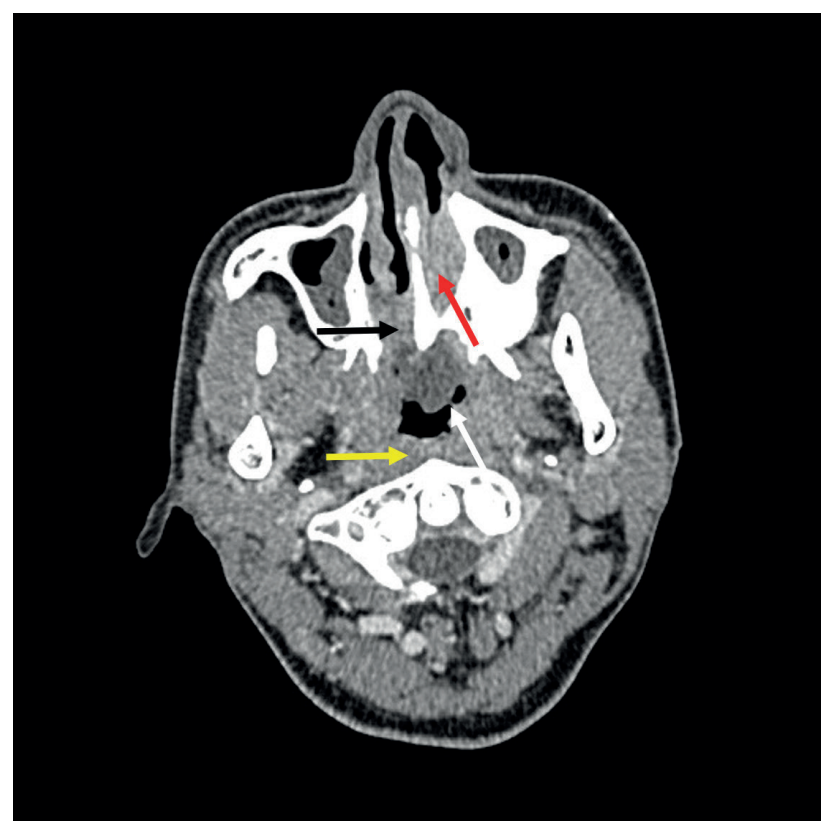

Figure 3. Axial CT scan of paranasal sinus showing soft tissue mass occupying posterior $2 / 3$ rd of the left nasal cavity (red arrow) and posterior $1 / 3$ rd of the right nasal cavity (black arrow), extending posteriorly to the nasopharynx (yellow arrow), inferiorly involving uvula and palate (white arrow).

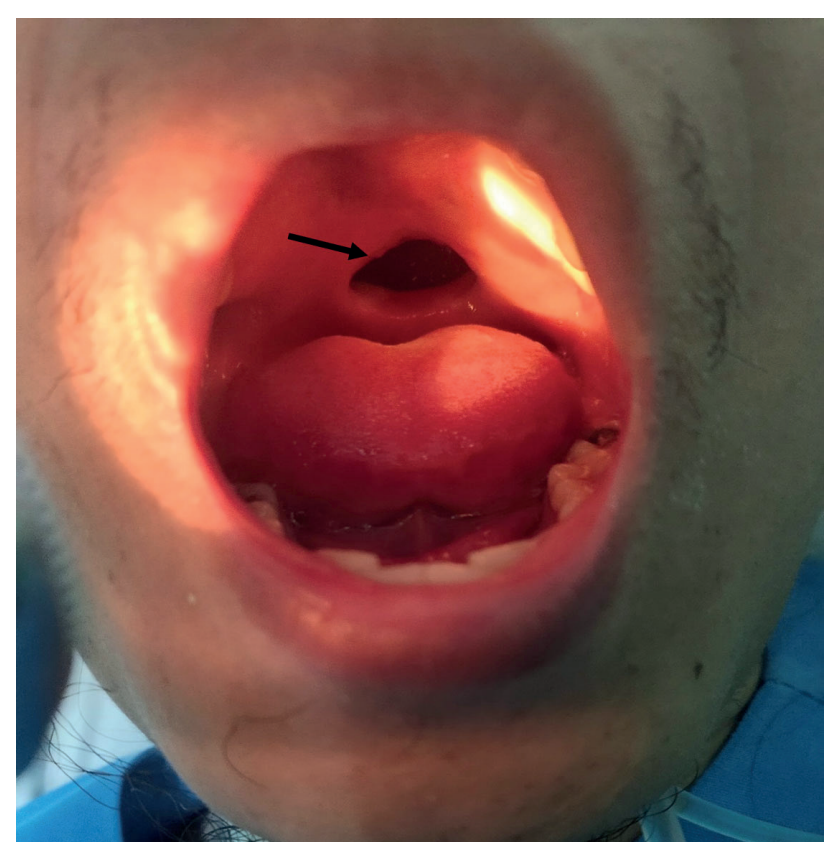

Figure 4. Oral cavity examination showed a smoothedge fistula measuring $5 \mathrm{~cm} \times 4 \mathrm{~cm}$ (black arrow).

between the oral and nasal cavity. Thus, it impairs both oral and nasal functions, such as swallowing and speech, through nasal regurgitation of food and nasal speech respectively, besides having the risk of nasal infection as a result of food lodgement. It causes hypernasality due to escape of air during speech and also leads to regurgitation of food and fluid into the nasal cavity [4]. There are several causative factors that may contribute to ONF formation (Table 1).

As for this patient, the most common differential diagnosis according to the sign and symptoms is left nasal squamous cell carcinoma (SCC), followed by nasopharyngeal carcinoma (NPC) and olfactory neuroblastoma (ONB). The reason is, from the patient's history, there is a progressive history of nasal blockage with epistaxis. The patients also complained of loss of appetite with significant weight loss. Examination of the left nasal cavity revealed a fleshy mass with ONF which is prone to the diagnosis of SCC as being the commonest sinonasal malignancy. However, due to the patient's age and no history of potential carcinogenic inhalant exposure, the diagnosis is unlikely. From examination as well, the patient has no palpable cervical nodes or neck swelling to suggest NPC. According to the gender predominance, ONB favours females more than males. Hence, hyposmia or anosmia is not a strong indicator for diagnosing an ONB and is non-specific. 
Table 1. Proposed causes of palatal perforation by Patil SR (6).

\begin{tabular}{|c|c|}
\hline Developmental & $\begin{array}{l}\text { Cleft palate (Secondary to maternal alcohol consumption and cigarette smoking, folic acid } \\
\text { deficiency, teratogenic drugs, viral infection, corticosteroid use and anticonvulsant therapy) }\end{array}$ \\
\hline Drug related & Narcotics (cocaine, heroin etc.,) \\
\hline Trauma & latrogenic, Thermal \\
\hline Infection & $\begin{array}{l}\text { Tertiary Syphilis, Tuberculosis, Leprosy, Typhoid, Mucormycosis, Actinomycosis, Aspergillosis, } \\
\text { Paracoccidioidomycosis, Histoplasmosis, Naso-Oral Blastomycosis, Leishmaniasis, Diphtheria, } \\
\text { Rhinoscleroderma }\end{array}$ \\
\hline Neoplasia & Lymphoma, Carcinoma, Melanoma, Acute Lymphoblastic Leukemia \\
\hline Collagen vascular disease & Wegener's granulomatosis, Systemic lupus erythematous \\
\hline Idiopathic & Midline lethal granuloma \\
\hline Granulomatous disease & Sarcoidosis, Crohns's disease \\
\hline Other & Rhinoliths \\
\hline
\end{tabular}

NKTCL has an ethnic predominance of East Asians and Latin Americans. It is associated with Epstein-Barr virus (EBV) infection, with preference for involvement of upper-aerodigestive-tracts, particularly nasal and paranasal areas $(80 \%$ of cases). It features a locally aggressive with a mix of necrotic cellular infiltrates and angioinvasion on histological examination. Males are more affected than females, with a median age of onset of 52 years [5]. Nonetheless, in this case report, it can also occur in young adults. The patient's initial symptoms are non-specific and include nasal obstruction with minimal epistaxis, which can mimic sinusitis symptoms. These symptoms usually precede ulceration and local destruction by months to years. However, the disease can have a rapidly progressive course, as in this patient, who presented with a relatively short history and significant weight loss, which is commonly presented in advanced cases of NKTCL. Similar to the less than half of cases reported, this patient also showed an increase in LDH levels with B symptoms as well.

NKTCL shows more local destructive behaviour as compared to other types of NHL. In this case, the initial ulcer over the soft palate was not in the midline, contrary to the commonly reported cases of NKTCL of maxillofacial or nasopharyngeal structures which tend to occur in the midline. The non-specificity of the symptoms of NKTCL based on the sites involved, therefore, may lead to delay in detection of the disease and a high probability of misdiagnosing with other conditions.

In the case of NKTCL, a diagnosis is not solely based on clinical manifestation. A combination of histopathology, imaging and even liver function parameters, i.e EBV PCR, LDH, are needed to confirm the diagnosis. The gold standard treatment for NKTCL is radiotherapy, but the treatment choice can be varied depending on the staging of the disease. Stage I and II are best treated with radiotherapy, chemotherapy or both, while stage III/IV is treated with the SMILE Protocol [6]. A novel regimen of steroids, i.e dexamethasone, methotrexate, ifosfamide, L-asparaginase, and etoposide (SMILE) showed promising remission in patients with NKTCL.

The ONF became wider despite day 19 of SMILE The protocol can be explained by the reduction of the inflamed and oedematous edges of the ONF as a direct positive effect of the treatment protocol. For this patient, if the ONF persists after completion of the treatment, it is indicated to surgically close the defect with primary closure. Local flap from buccal pad of fat or palatal flap can be incorporated if unable to achieve primary closure. If surgical closure fails, a maxillary obturator prosthesis may be applied [7].

As for the patient's follow-up, it is recommended to have a multidisciplinary team involving hemato-oncology, oromaxillofacial surgery, and otorhinolaryngology outpatient clinic to closely monitor the patient's progress and tumour surveillance [8].

\section{CONCLUSION}

Diagnosis of a rare NKTCL as the cause of ONF is challenging. Hence, one must have a high index of 
suspicion with several differential diagnoses when encountering such cases. It is essential to involve a multidisciplinary team to help diagnose and treat NKTCL, especially in cases presenting with non-specific nasal and oral symptoms. The disease should be staged appropriately to help in deciding the best treatment protocol. Furthermore, the main presentation of this case was an ONF, which by itself is a rare complication of NKTCL.

\section{CONFLICT Of INTEREST}

The authors declare no conflict of interest..

\section{ree REFERENCES Cen}

[1] Sarıbacak Can E, Okutan H, Albayrak M, et al. Extranodal natural killer/T-cell lymphoma: A rare nasal-type case. Turk J Haematol 2016; 33: 74-75.

[2] Patil SR. Proposed classification for the palatal perforation. J Interdiscipl Med Dent Sci. 2016; 4(2): 192.

[3] Bhatt VR, Koirala B, Terjanian T. Extranodal natural killer/T cell lymphoma, nasal type presenting as a palatal perforation and naso-oral fistula. BMJ Case Rep. 2011; 10: 1-4.

[4] Inman DS, Thomas P, Hodgkinson PD, et al. Oro-nasal fistula development and velopharyngeal insufficiency following primary cleft palate surgery - An audit of 148 children born between 1985 and 1997. Br J Plast Surg. 2005; 58(8): 1051-1054.
[5] Wu XC, Andrews P, Chen VW, et al. Incidence of extranodal non-Hodgkin lymphomas among whites, blacks, and Asians/Pacific Islanders in the United States: Anatomic site and histology differences. Cancer Epidemiol. 2009; 33(5): 337-346.

[6] Tse E, Kwong YL. The diagnosis and management of NK/Tcell lymphomas. J Hematol Oncol. 2017; 10: 85.

[7] Kaur P, Kaur J. Oronasal fistula and complete edentulism: What to Do? Dent J. 2014; 2(4): 142-154.

[8] Ciftciler R, Cifciler AE, Saglam EA, et al. Follow-up of patients with atypical lymphoproliferation in bone marrow or lymph node biopsy. Acta Medica. 2021; 52(1): 37-42. 